\title{
Whole Health System Change in Ireland: A Collaborative Inquiry Into the Enablers and Challenges of Implementation
}

\author{
Aine Carroll ( $\square$ aine.carroll@ucd.ie) \\ University College Dublin https://orcid.org/0000-0002-4383-8650 \\ Valerie Twomey \\ National Rehabilitation Hospital
}

\section{Research article}

Keywords: Whole health system change, Multi-Stakeholder Engagement Process, Collaborative Inquiry, enablers, challenges, implementation, clinical programme, World Café, Thematic analysis

Posted Date: September 21st, 2020

DOl: https://doi.org/10.21203/rs.3.rs-62405/v1

License: (c) (i) This work is licensed under a Creative Commons Attribution 4.0 International License.

Read Full License 


\section{Abstract}

\section{Background}

Across the world, health and care systems are struggling to contain costs and improve quality of services by improving prevention, developing wellness programmes, and providing person-centred coordinated health and care. The development of the National Clinical Programmes (NCPs) in Ireland in 2009 coincided with a profoundly challenging period for the Irish economy and consequently for publicly funded health services. These programmes are focused on implementing clinical and operational models of care that have access, quality and value at their core. By 2012, there was a clear recognition among senior health leaders that the desired outcome of nationalising best practice and achieving whole system change had not been achieved through the NCPs and a series of world café events were then planned to assess the challenges that had been experienced by the programmes in order to inform the next phase.

Methods

A Multi-Stakeholder Engagement Process using the World Café method of collaborative participatory inquiry, was conducted to obtain a rich, in-depth understanding of the development and implementation of health service reform in Ireland. Three keys questions were explored through an inductive approach to analysis of themes that emerged from conversations with key stakeholders.

Results

There were 59 participants. A total of 408 responses were received. Using an inductive approach to analysis of themes 5 key themes emerged; Integrated care; Patient Centred Care; Clinical Governance; Funding models and e-Health.

\section{Conclusion}

Having a clear vision, a prescribed methodology and clinician buy and involvement in the design of new models of care is not sufficient to ensure implementation. A whole system approach to the design and implementation of new models of care must be supported by essential enablers such as policy, financial and human resource models and knowledge management systems. This research informed the planning and design for the second phase of the programmes and sought to overcome the barriers and work with operations and policy makers to improve outcomes for patients by implementing person centered evidence-based models of integrated care.

\section{Background}

Across the world, healthcare service delivery seeks to control costs and improve quality of services for all by increasing access to preventive services, wellness programmes and continuous medical care (1). The HSE (Health Service Executive) National Clinical Programmes (NCPs) in Ireland are a series of clinically led quality improvement initiatives that were established by the Irish health system in 2009. They were 
initiated in response to a growing awareness of inadequate implementation of clinical standards and regional variation in practice revealed by a series of inquiries into serious adverse events in Irish hospitals during the early 2000 s $(2,3)$.

The National Clinical Programmes are a joint initiative and have formed a powerful guiding coalition through a partnership between HSE and Clinicians through the Forum of Postgraduate training colleges, the Irish Association of the Directors of Nursing and Midwifery and the Therapy Professions Committee and most importantly, in partnership with patients. The programmes are focused on implementing models of care that simultaneously seek to pursue improving access, quality and cost-efficiency. Although the approach to achievement of these goals was very much at the discretion of each individual programme, common aspects across the programmes include: (1) a defined governance structure (2) detailed description of how cost savings would be made, (3) development of care pathways and models of care, and (4) emphasis on measuring success based on metrics. Each programme has a governance structure involving the HSE, the professional body and clinicians. A Clinical Advisory Group (CAG) consisting of a diverse group of clinicians in the specialty is appointed by the professional body and a National Clinical Lead. The CAG provides clinical strategic planning and direction, reviews and agrees practice guidelines and models of care and supports and facilitates the implementation of the nationally agreed model of the programmes. In addition, each programme has a National Programme Manager, Regional Clinical Leads and a Multidisciplinary Working Group established in order to support the implementation of the work of the programme locally. The NCPs are supported by a professional Diploma in Quality Improvement in association with the Royal College of Physicians of Ireland. Many of the national clinical leads of the NCPs have undertaken this diploma along with key members of their clinical and management teams. Part of the emphasis within the diploma and in the NCPs is on standardisation as an element of quality improvement.

The adoption of the NCPs coincided with a profoundly challenging period for the Irish economy and consequently for publicly funded health services. Very large funding and staffing cuts have been imposed on the Irish health system since 2009. Despite this, evidence from several quality metrics indicates that services have been maintained or enhanced since the cuts. However, after 3 years, despite the improvements indicated above, there was clear recognition that the desired outcome of nationalising best practice and achieving whole system change had not been achieved and a series of engagement events were then planned to assess the challenges that had been experienced by the programmes in order to inform the next phase of the improvement initiatives.

\section{Methods}

A Multi-Stakeholder Engagement Process utilising the World Café method of participatory inquiry $(4,5)$, was conducted with 59 key stakeholders involved in the Clinical Programmes including clinicians, managers, academics and patients to access the participants' experience and expertise of the Clinical Programmes to date to inform phase 2. An inductive approach using qualitative thematic analysis of the written material was used to determine themes for identification. This was chosen as it provides a flexible 
and useful research methodology, which can potentially provide a rich and detailed, yet complex account of data obtained. Participants were required to respond to 3 key questions presented during the World Café and to write their responses verbatim for submission to the investigators. Questions were designed with health system redesign in mind to elicit responses that would capture the participants' subjective experiences of the healthcare system regarding the implementation the clinical programmes.

\section{Participants}

Participants were either national clinical leads, GP leads, programme managers, and/or members of the postgraduate medical training forum. Participants also included key stakeholders such as patients and patient advocacy organisations and representatives from academic institutions. At the time of the study, all participants were actively engaged in quality improvement work with the National Clinical Programmes.

\section{Procedure}

Purposive sampling was used to identify participants. This method was chosen because the programmes are very diverse and heterogeneous, and it was felt that participants needed to reflect the breadth of the programmes without too many participants being involved to make the running of the events and meaningful dialogue difficult. Identified participants were then invited to participate by a letter from the National Director. 2 world café events were held.

\section{Conduct}

Developed by Juanita Brown and David Isaacs the World Café is a participatory method that seeks to facilitate change by bringing together key stakeholders of a system of interest and using a highly structured movement process, enables evolutionary conversations that generates collective discoveries that are gathered and shared $(4,5)$. This approach recognises that answers to difficult questions often exist in the collective knowledge of networks of individuals with a shared interest. It was designed as a means of cultivating and harnessing the power of 'conversations that matter' and follow seven core design principles: (6)

1. There must be a clear purpose and parameters in which collaborative learning will develop.

2. The event must be hosted in a hospitable space

3. It should explore questions that matter to participants.

4. Every participants contribution is encouraged and valued

5. It should facilitate cross-pollination and connect diverse viewpoints.

6. There should be collective listening to identify patterns, insights and discoveries.

7. The collective discoveries are harvested and shared.

The objective of the sessions was to obtain the views of the invited participants on the following three questions which were consciously orientated towards the principles of appreciative inquiry and developed 
as 'provocative propositions' (7):

1. What is good about the Clinical Programmes?

2. What is not good about the Clinical Programmes?

3. Make one suggestion on how to improve the Clinical Programmes

The café sessions considered each of the three questions in turn.

The World Café setting created a relaxed environment where participants were grouped at tables of 6-8. A draw was used for the allocation of tables.

Only one question was presented at a time. Every table "chatted" about the question and when doing so jotted down their comments on the cards provided - one card per comment.

30 minutes per question was allocated.

\section{Data collection}

At the end of each session the cards were collected. No names or table numbers were on the cards so that feedback was totally anonymous.

The feedback comments were entered in an excel spreadsheet for analysis and reporting. The themes were not identified prior to examination of the comments. Only as the comments were analysed did the themes come together.

\section{Ethical Approval and Consent to Participate}

This activity was conceived as a collaborative inquiry with the primary purpose of developing a shared understanding of challenges to implementation to inform the next phase of the programmes. The activity was screened in accordance with HSE Research Ethics guidance and was deemed not to require research ethics committee review. The work observed the ethical principles articulated within the Belmont Report (8)

All written comments and notes were captured anonymously. Verbal consent was obtained from participants at the start of the events. Participants were informed in the invitation and at the start of the meeting that the written notes would be collected and analyzed and used to create a report from the activity. Participants were informed that they would have access to a draft report for their feedback prior to the wider circulation of the report.

\section{Data Analysis}

The 6 phases of thematic analysis according to Braun and Clarke were observed (9). Three investigators analysed the data. The investigators familiarised themselves with the data by reading and rereading the written submissions and noting down initial ideas. Codes were initially given to certain recurring words or 
ideas. These codes were then simplified and expanded into interpreted categories and themes as patterns in the data emerged. The themes were then expanded on and further revised. The final stage was to refine themes and define why they were interesting and what was interesting about them. Finally, a report was produced.

\section{Results}

In-depth analysis of responses resulted in a significant number of initial codes, which were further analyzed to a set of final codes and then categories. These categories were evident across majority of participants. The process is summarized in Figure 1.

Insert Figure 1. Codes, Categories and Emergent Themes

\section{Question 1: What is good about the National Clinical Programmes?}

A total of 408 responses were received. These responses were grouped into initial codes as shown in figure 2 .

Insert Figure 2: Codes Identified from what is good about the National Clinical Programmes

Question 2: What is not good about the National Clinical Programmes?

A total of 451 responses were received for question 2 . These responses were grouped into the following 23 codes as shown in figure 3 .

Figure 3: Key codes from question 2: What is not good about the national clinical Programmes? Insert figure 3: Key codes from question 2: What is not good about the national clinical Programmes? Question 3: Make one suggestion on how to improve the National Clinical Programmes

A total of 71 comments were received. These comments were grouped into the following 12 codes as shown in figure 4.

Insert Figure 4: Key themes from question 3: Make one suggestion on how to improve the Clinical Programmes

\section{Emergent themes:}

\section{Theme 1: Integrated care}

Participants described the importance of a partnership approach to the Clinical Programmes. One comment stated, "College involvement gives the NCPs credence and credibility" with another commenting that the multidisciplinary nature of the programmes "Harbour better understanding between the various 
disciplines and health settings". There were many comments on the lack of integration across the programmes with one comment being "The programmes are at risk of becoming silos"

\section{Theme 2: Patient centred}

Participants described the importance of patient centredness. One comment stated that "It's the right thing to do" and that there is a "Sincere genuine interest in patients". Other comments stated that the NCPs offered the opportunity to "Identify the real priorities vs political priorities" and that "Patient and patient groups must be included in all stages of health planning".

\section{Theme 3: Clinical Governance}

Participants described the importance of clear Clinical Governance. And one commented that currently that "wasn't clear". Another comment stated that there is a need for "proper governance to get national policy Programmes implemented" and another comment was that there was a "Lack of evaluation of impact of change".

\section{Theme 4: Funding Model}

Participants described the need for clear funding models to support the work of the Clinical Programmes. One representative comment was that there was "In-sufficient funding" and another that participants "Cannot secure money that is saved" and another that there was "No autonomy re spend".

\section{Theme 5: eHealth}

Participants described severe challenges with Information and communications technology (ICT) and procurement with one representative comment stating "ICT procurement and approvals process is very slow and cumbersome even for relatively small project and for larger Programmes - directorate approval" A sense of frustration was evident form the comments and one comment highlighted the "Need for better national records / registers and information technology in general".

\section{Discussion}

How to successfully engage with key stakeholders was a big challenge at this stage of development of the Clinical programmes. The country was in deep recession and the initial enthusiasm that new initiatives have, had started to wane. The importance of successful engagement was identified as a major priority to sustain involvement and participation and to harness the collective knowledge existing within the clinical programmes.

The experience of the facilitators and participants was very positive and showed that the World café approach was successful and allowed the participants to make sense of how they perceived themselves as participants in the clinical programmes, in relation to colleagues, clinical and non-clinical and what resources and systems needed to be in place to support the next phase of the programmes. 
This approach facilitated the development of a new experience in engagement and knowledge generation; a conversational process that helped the participants to engage in constructive dialogue around matters of importance to them, to build social networks and to foster collaborative learning. In relation to other participatory and collaborative approaches, the World Café was powerful in terms of cross-pollination of ideas and perspectives and the connection of diverse viewpoints into a coherent vision. The sensemaking that took place through the highly structured process, yet in a relaxed nonthreatening environment, enabled co-evolutionary creative conversations that generated discoveries that were harvested and shared.

While the considerable achievements of the programmes were clear, analysis of the themes reveals the essential need to maintain and enhance clinical leadership, develop clinical pathways that are truly patient-centred, and seamlessly cross organisational and professional boundaries. There is a recognized requirement to; align programme design with service priorities, enhance evidence-base and performance and outcome measurement, ensure structured and consistent implementation and ensure alignment with key enabling functions such as Finance, Human Resources (HR) and ICT. The output from this participatory inquiry supported the development of the Integrated Care programmes which are now underway. These integrated care programmes are now playing a key role in improving patient care and are a key pillar of national health policy.

\section{Limitations}

There is very little in the scientific literature about using a World Café approach in healthcare however, we feel that this method fits well within the action research family of approaches as it relies on an appreciation of local knowledge and co-design and co-evolution of solutions (10)

\section{Conclusion}

The World Café method encouraged purposeful, collaborative engagement with the heterogeneous groups of stakeholders involved in the clinical programmes The approach allowed a collective and collaborative sensemaking of the enablers and barriers to change in the health system and allowed a coproduction of next steps. It was recognized that having a clear vision, a prescribed methodology and clinician buy and involvement in the design of new models of care is not sufficient to ensure implementation. For new models of health and social care to be successful, a whole system approach to design and implementation must be taken, adequately supported by key enablers including; policy, financial and HR models and knowledge and information management systems. The World Café approach proved to be a very powerful method of engagement that facilitated a collective intelligence about the challenges of implementation from many different perspectives and allowed the co-creation of solutions to inform the next phase of the programmes.

\section{Abbreviations}


CAG: Clinical Advisory Group

HR: Human resources

HSE: Health Service Executive

ICT: Information and communications technology

NCP: National Clinical Programmes

\section{Declarations}

\section{Ethics approval and consent to participate:}

This work did not require formal ethics approval in accordance with the national guidelines provided by HSE Research Ethics guidance (11)

No personal data was recorded, and data was anonymised. The work is GDPR compliant.

\section{Consent to participate:}

All participants were informed that the output from the events would be recorded and reported and all participants provided verbal consent. All participants were informed that analysis of anonymous responses would be used in a variety of reports for learning and research purposes.

\section{Availability of data and materials:}

The datasets used and/or analysed during the current study are available from the corresponding author on reasonable request.

\section{Competing interests:}

At the time of the study, Prof Carroll was National Director of Clinical Strategy and Programmes in the HSE. Dr Twomey was previously a Senior Programme manager.

\section{Funding:}

No funding was received

\section{Authors' contributions}

$A C$ and VT contributed to the design of the study. Staff within the office were responsible for data acquisition. VT and others were responsible for analysis and interpretation of the data. AC and VT were responsible for the drafting of the manuscript and all critical revisions. All authors agree to be accountable for all aspects of the work ensuring that questions related to the accuracy or integrity of any 
part of the work are appropriately investigated and resolved. All authors read and approved the final manuscript.

\section{Acknowledgements}

The authors wish to acknowledge Ms Sarah McCormack who contributed towards the organisation of the world café events and data collection and all the participants who gave freely of their time and experience.

An abstract of a presentation on this work presented at the International Conference on Integrated care 2017 was published: Carroll Á, White B, Twomey V. Whole health system change in Ireland: Analysis of the challenges of implementation. International Journal of Integrated Care. 2017;17(5): A402. DOI: http://doi.org/10.5334/ijic.3721

\section{References}

1. Berwick DM, Nolan TW, Whittington J. The Triple Aim: Care, Health, And Cost. Health Affairs. 2008;27(3):759-69.

2. Clark MH. The Lourdes hospital inquiry: an inquiry into peripartum hysterectomy at Our Lady of Lourdes Hospital, Drogheda: Stationery Office; 2006.

3. McDaid D, Wiley M, Maresso A, Mossialos E. Health systems in transition Ireland: health system review. 2009.

4. Brown J. The World Café: Living knowledge through conversations that matter: Fielding Institute; 2001.

5. Brown J, Isaacs D. The World Café: Living knowledge through conversations that matter, The Systems Thinker, 12, 1-5. 2001.

6. Fouché C, Light G. An Invitation to Dialogue: 'The World Caféln Social Work Research. Qualitative Social Work. 2011;10(1):28-48.

7. Reed J. Appreciative inquiry: Association for Talent Development; 2006.

8. Health UDo, Services H. The Belmont Report: Office of the Secretary, Ethical Principles and Guidelines for the Protection of Human Subjects of Research, the National Commission for the Protection of Human Subjects of Biomedical and Behavioral Research. Washington: Department of Health, Education, and Welfare; 1979.

9. Braun V, Clarke V. Using thematic analysis in psychology. Qualitative research in psychology. 2006;3(2):77-101.

10. Greenwood DJ. Action research: Unfulfilled promises and unmet challenges. Concepts and transformation. 2002;7(2):117-39.

11. HSE. Research Ethics Committees https://www.hse.ie/eng/services/list/5/publichealth/publichealthdepts/research/rec.html2020 


\section{Figures}

Preliminary Codes

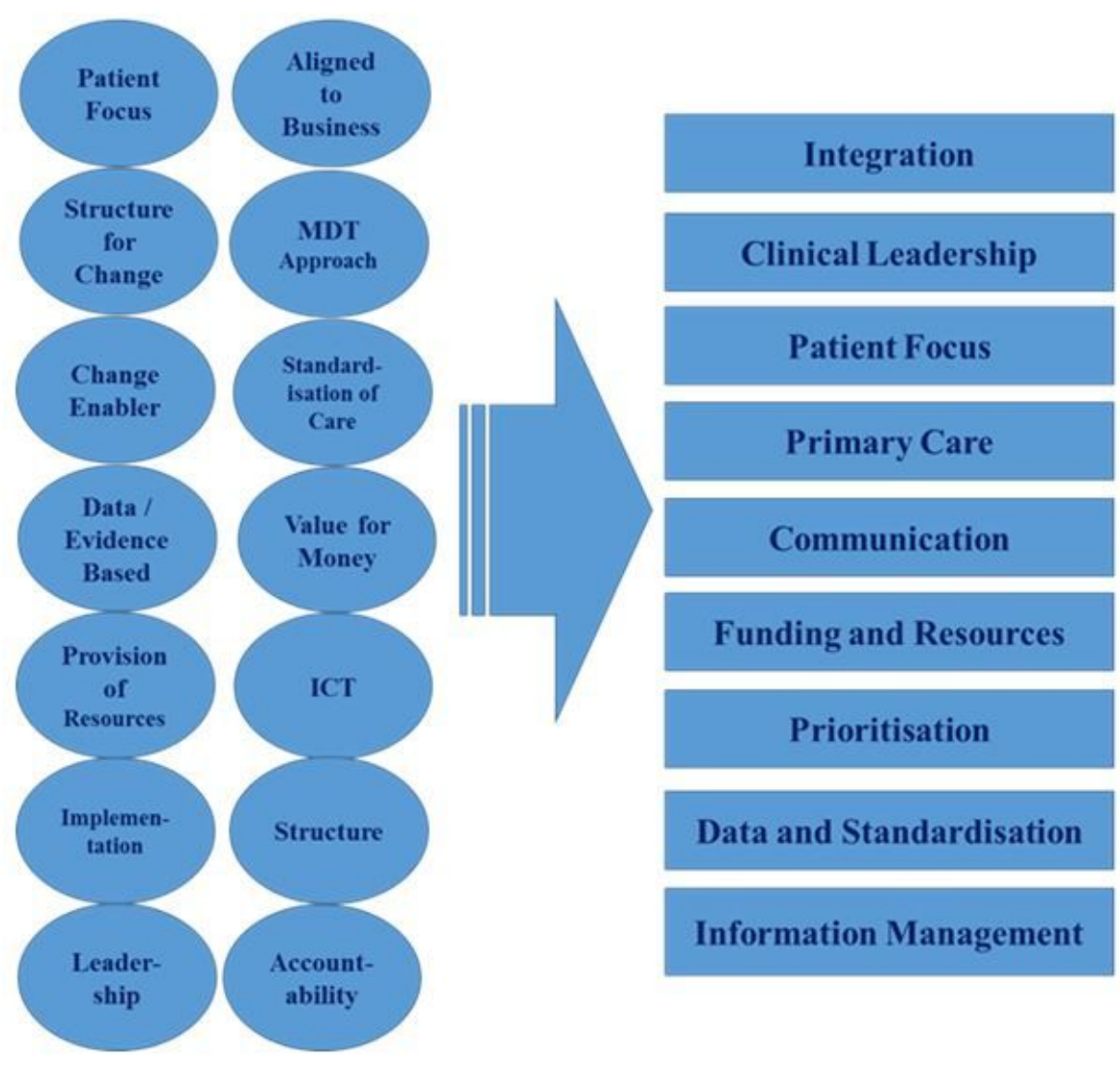

Themes

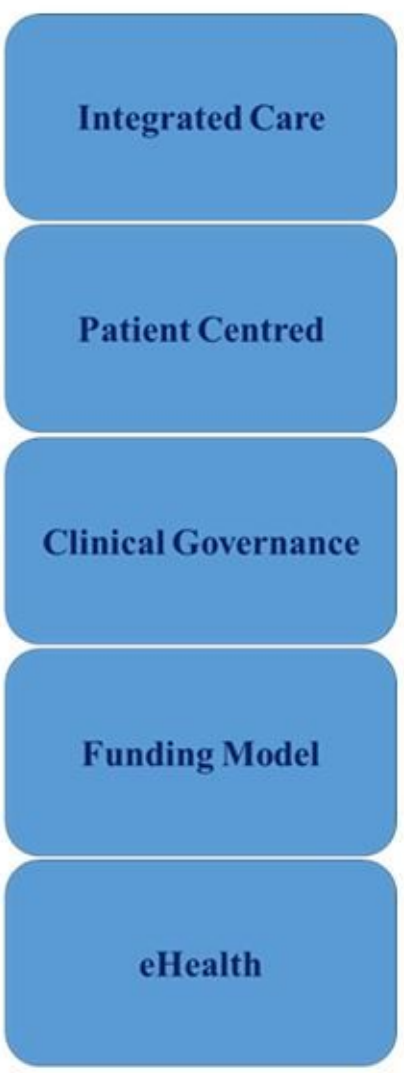

\section{Figure 1}

Codes, Categories and Emergent Themes 


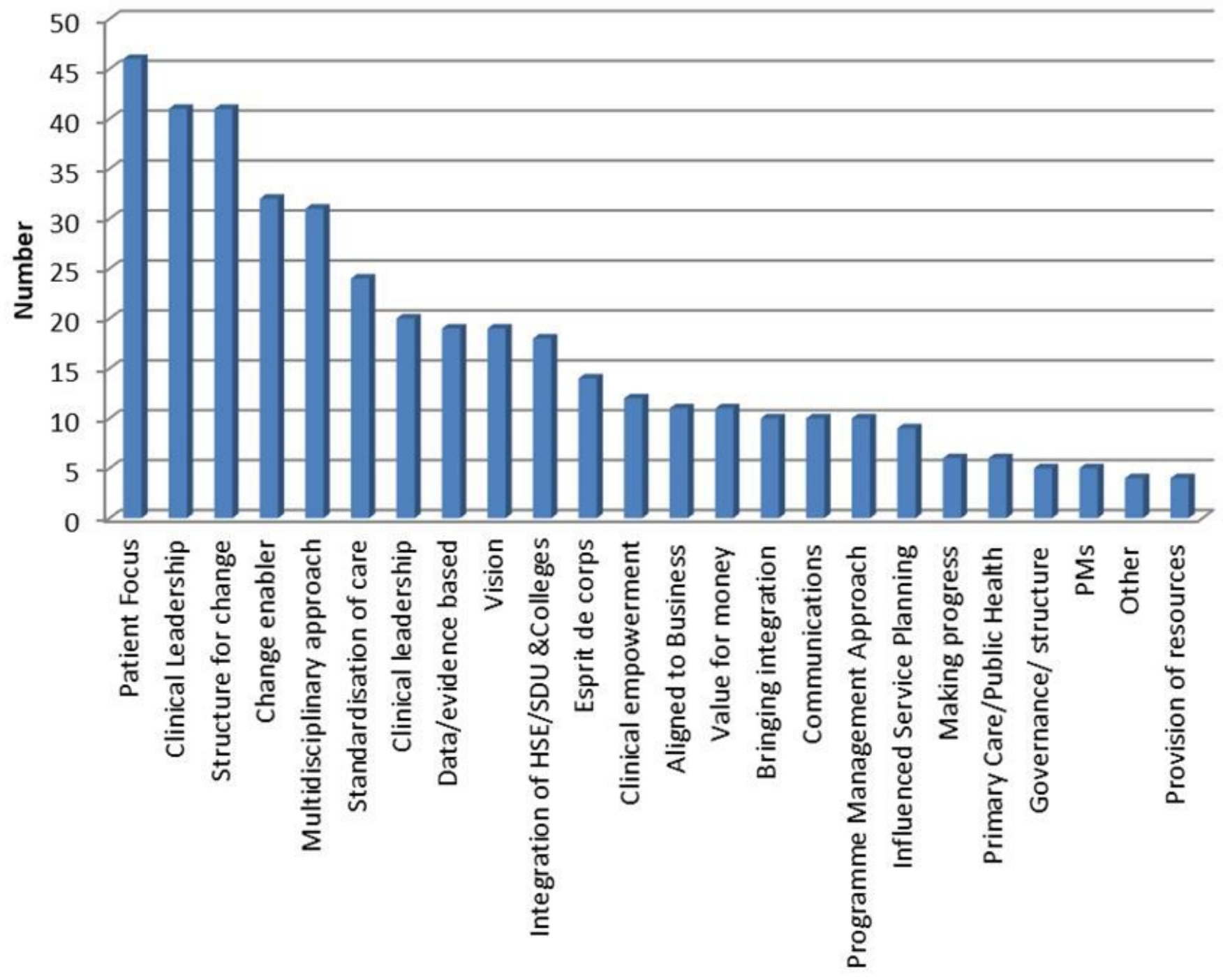

Figure 2

Codes Identified from what is good about the National Clinical Programmes 


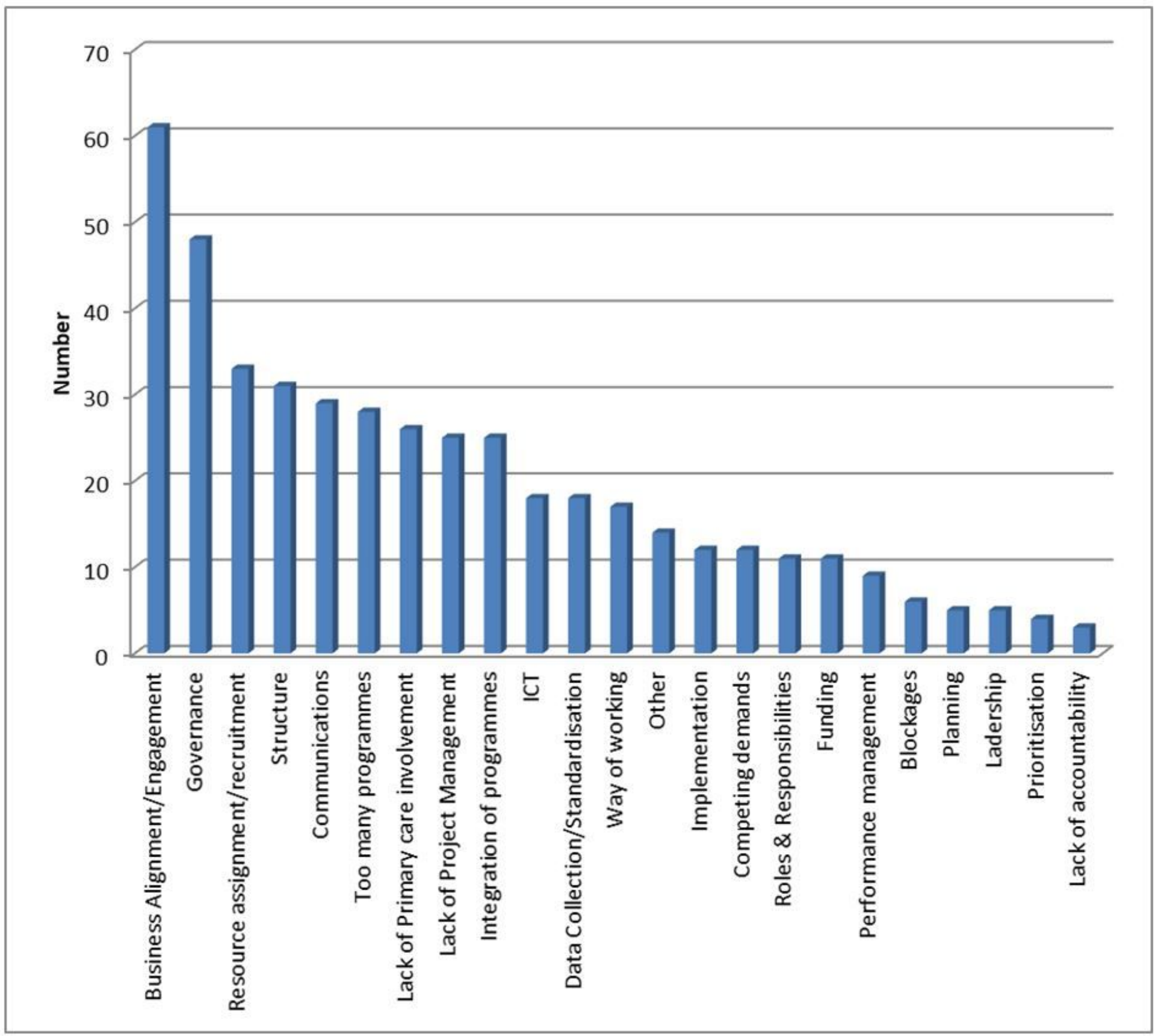

Figure 3

Key codes from question 2: What is not good about the national clinical Programmes? 


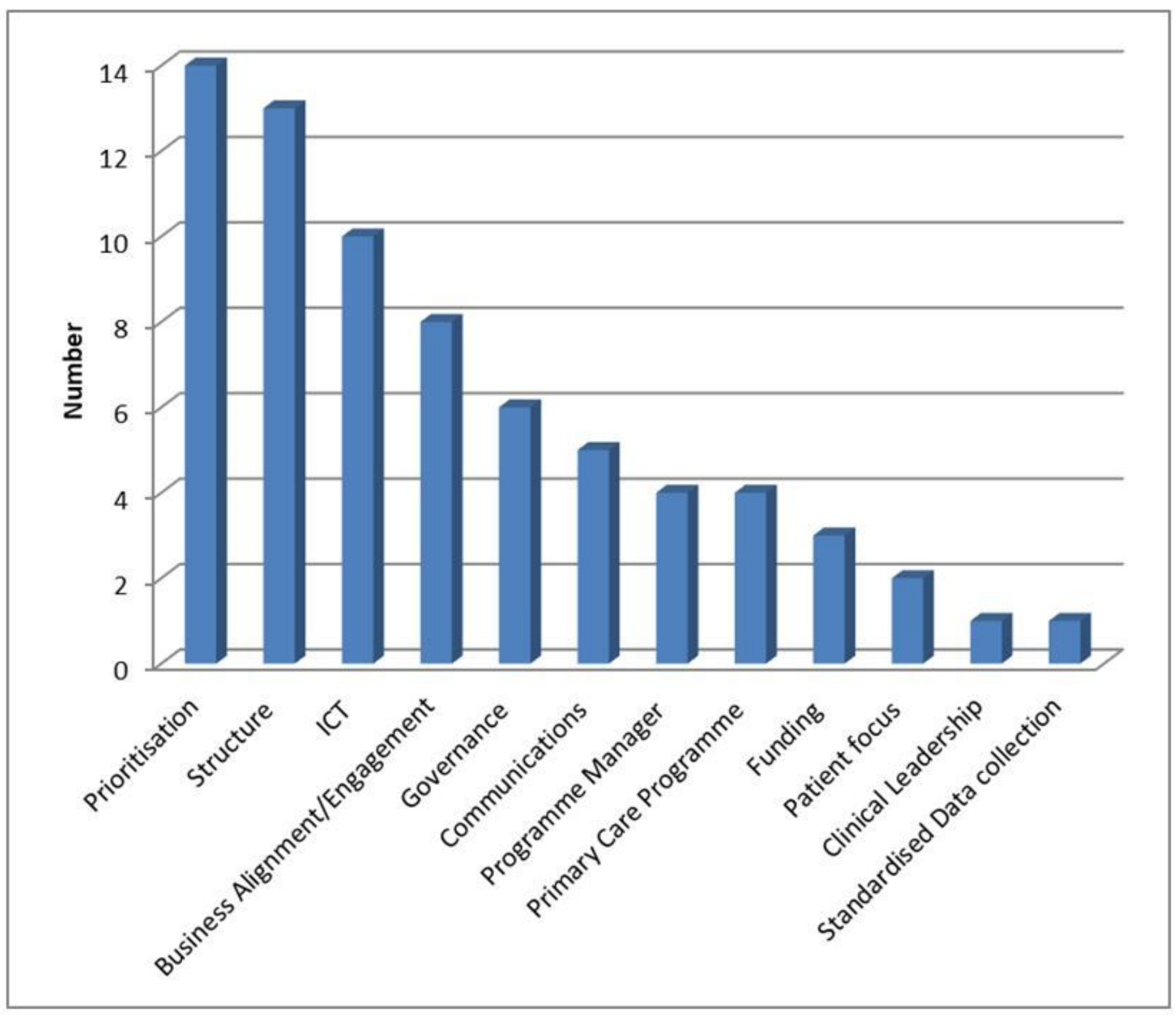

Figure 4

Key themes from question 3: Make one suggestion on how to improve the Clinical Programmes 